\title{
MÉTODO MULTIRRESÍDUOS PARA ANÁLISE DE 240 AGROTÓXICOS EM SOLOS DO PLANTIO DE TOMATE POR CROMATOGRAFIA LÍQUIDA DE ULTRA DESEMPENHO ACOPLADA À ESPECTROMETRIA DE MASSA
}

\author{
ARTIGO ORIGINAL \\ MAZZEI, João Roberto Fortes ${ }^{1}$ \\ FREIRE, Estevão ${ }^{2}$ \\ SERRA, Eduardo Gonçalves ${ }^{3}$ \\ MACEDO, José Ronaldo de ${ }^{4}$ \\ OLIVEIRA, Angélica Castanheira de ${ }^{5}$ \\ BASTOS, Lucia Helena Pinto ${ }^{6}$
}

1 Doutorado em andamento em Engenharia Ambiental. Mestrado em Mestrado Profissional em Engenharia Ambiental. Especialização em Metodologia do Ensino de Química. Graduação em Química.

2 Orientador. Doutor em Engenharia pelo Programa de Engenharia de Minas, Metalúrgica e de Materiais da Universidade Federal do Rio Grande do Sul.

3 Orientador. Doutor em Engenharia Oceânica pela Coppe/UFRJ; Professor Associado da Escola Politécnica da Universidade Federal do Rio de Janeiro, e PróReitor de Graduação da UFRJ.

${ }^{4}$ Orientador. Doutor em Ciências pelo Centro de Energia Nuclear na Agricultura / CENA - da Universidade de São Paulo.

${ }^{5}$ Mestre em vigilância sanitária em saúde (FIOCRUZ/INCQS).

${ }^{6}$ Doutora em vigilância sanitária em saúde (FIOCRUZ/INCQS). 
CARDOSO, Maria Helena Wohlers Morelli ${ }^{7}$

MAZZEI, João Roberto Fortes. Et al. Método Multirresíduos para Análise de $\mathbf{2 4 0}$ Agrotóxicos em Solos do Plantio de Tomate por Cromatografia Líquida de Ultra Desempenho Acoplada à Espectrometria de Massa. Revista Científica Multidisciplinar Núcleo do Conhecimento. Ano 06, Ed. 01, Vol. 08, pp. 34-67. Janeiro de 2021.

ISSN:

2448-0959,

Link

de

acesso: https://www.nucleodoconhecimento.com.br/engenharia-ambiental/metodomultirresiduos

\section{RESUMO}

Neste trabalho, otimizou-se um método analítico para a determinação de resíduos para os agrotóxicos-foco: Azoxistrobina, Boscalida, Carbendazim, Clorantraniliprole, Clotianidina, Diafentiuron, Difenoconazol, Dimetomorfe, Espinetoram, Espinosade A, Espinosade D, Fenuron, Imidacloprido, Indoxacarbe, Metalaxil M, Metoxifenozida, Tiametoxan em solo derivado do plantio do tomate, com o objetivo de comparar os níveis de contaminação desses compostos em amostras de solo. Foi utilizado o método de extração QuEChERS modificado e Cromatografia Líquida de Ultra Desempenho acoplada à Espectrometria de Massas Sequencial, com fonte de ionização por Eletronebulização no modo ESI (+/-). O método consistiu na extração de $15,0 \mathrm{~g}$ de solo com $15 \mathrm{~mL}$ de solução saturada de hidróxido de cálcio pH 12,3 e 15 $\mathrm{mL}$ de acetonitrila, com consequente partição em efeito "salting out" através de $6,0 \mathrm{~g}$ de sulfato de magnésio anidro e 1,5 $\mathrm{g}$ de cloreto de sódio. As fases foram separadas por centrifugação a $3700 \mathrm{rpm}$ por $7 \mathrm{~min}$. Os extratos foram diluídos com MeOH grau licrossolv ${ }^{\circledR}$ e injetados em cromatógrafo. $O$ método foi validado com base nos parâmetros de linearidade, LOD, LOQ, precisão e exatidão. Linearidade entre 0,2 e $20,0 \mu \mathrm{g} \mathrm{L}^{-1}$, coeficientes de determinação maiores que 0,99 . Os valores de LOQ para o método foram $13 \mu \mathrm{g} \mathrm{kg}^{-1}$ para Espinosade e $7,0 \mu \mathrm{g} \mathrm{kg}^{-1}$ para os demais pesticidas. O método apresentou boa precisão, com valores de RSD $<20 \%$, e exatidão, com

${ }^{7}$ Doutora em vigilância sanitária em saúde (FIOCRUZ/INCQS). 
recuperações entre 70 e 120\% para a grande maioria dos compostos analisados. As curvas analíticas foram preparadas com extratos de solo branco de referência, de forma a minimizar o Efeito Matriz. O método foi considerado adequado para a análise de resíduos de agrotóxicos em solo, uma vez que satisfaz aos parâmetros de validação de métodos cromatográficos (European Comission, 2018). Depois da validação, o método foi utilizado para análise de resíduos destes agrotóxicos em amostras de solo provenientes dos plantios de tomate convencional, orgânico e sustentável. Tornando possível comparar os níveis de impactos ambientais gerados. Além de validar o método analítico para os agrotóxicos-foco do estudo conseguiu-se também a validação para mais 240 compostos, entre autorizados e não autorizados para a utilização no plantio do tomate.

Palavras-chave: Contaminantes em solos, resíduos de agrotóxicos, QuEChERS, UPLC-MS/MS.

\section{INTRODUÇÃO}

O cultivo do tomate é suscetível ao surgimento de insetos-praga e doenças. Dados da pesquisa de Carvalho (2017) citam que o cultivo do tomate é vulnerável ao acometimento de doenças causadas por insetos-praga, sendo a mosca branca (Bemisia), uma das principais pragas que acometem o tomate. A Bemisia argentifolii e a Bemisia tabaci, são as duas principais espécies de mosca-branca responsáveis por causar prejuízos para o cultivo do fruto. No trabalho do autor, foram entrevistados tomatecultores do Município de Cambuci (Rio de Janeiro), em que foi observado que cerca de $60 \%$ dos plantadores realizam até duas aplicações de agrotóxicos semanalmente. Em $2 \%$ dos casos, os plantadores descreveram que, dependendo da situação, como por exemplo: se surgirem doenças ou o tempo estiver chuvoso, há necessidade de maior número de aplicações, podendo chegar a três vezes por semana.

Morfologicamente não há diferença entre as duas espécies. Porém, a primeira é mais agressiva, uma vez que, além de apresentar maior resistência às condições adversas 
do ambiente e a alguns agrotóxicos convencionais, apresenta maior índice de reprodução, acomete maior número de plantas hospedeiras e consegue completar todo o seu ciclo de vida no tomateiro. Por esse motivo, é necessária a utilização de variados agrotóxicos para o controle dessas e outras pragas que acometem a plantação do tomate (ESALQ, 2017).

Neste trabalho, optou-se por utilizar a terminologia orientada pela legislação brasileira - agrotóxicos - por considerar que esse termo, apesar de não cobrir na essência todos os produtos utilizados, engloba o maior número de atributos necessários à descrição das substâncias que compõem esse universo e agrega mais transparência e ética para o leitor, o usuário e o consumidor dos produtos em que tais compostos são utilizados (SOBER, 2018).

Segundo a revisão da legislação brasileira sobre agrotóxicos publicada em 28/06/2018 (MAPA, 2018):

O termo agrotóxico não é utilizado por nenhum outro país ou organização internacional que trata do tema. A Comissão do Codex Alimentarius, organização internacional de referência para alimentos no Acordo sobre a Aplicação de Medidas Sanitárias e Fitossanitárias da Organização Mundial do Comércio (OMC), utiliza o termo em inglês e francês "agrotoxic" e em espanhol "plaguicida". Dessa forma, é preciso alterar o termo pesticida para agrotóxico, a fim de alinhar a legislação brasileira às práticas internacionais.

O tomate foi escolhido como objeto desse estudo por conta das características específicas que apresenta, isto é, plantações como a do tomate (Solanum

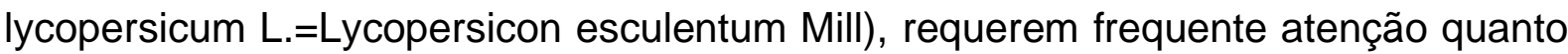
à infestação de pragas e ervas daninhas, o que implica na necessidade de aplicação de agrotóxicos com grande frequência.

Ao entrar em contato com o solo, os agrotóxicos estão sujeitos aos processos físicoquímicos que propiciam agravamento de sua ação no ambiente. Devido à necessidade de uso racional dos insumos agrícolas para minimizar os impactos ambientais da agricultura, muitos estudos têm sido realizados com o objetivo de 
compreender o comportamento desses produtos no solo. No entanto, pouco se sabe sobre o comportamento desses agrotóxicos em solos tropicais.

\section{CONTAMINAÇÃO DE SOLOS POR AGROTÓXICOS}

De acordo com os estudos de Azevedo (2018), mesmo com maior controle de aplicação dos agrotóxicos, o solo é o destino final dos produtos químicos usados na agricultura, sejam eles aplicados diretamente no solo, na parte aérea das plantas ou até mesmo nos frutos ensacados. Ao entrar em contato com o solo, os agrotóxicos e herbicidas estão sujeitos a processos físico-químicos que propiciam agravamento de sua ação no ambiente. Segundo o autor, devido à necessidade de uso racional dos insumos agrícolas para minimizar os impactos ambientais da agricultura, muitos estudos têm sido realizados com o objetivo de compreender o comportamento desses produtos no solo.

O solo funciona como filtro, retendo muitas das impurezas que nele são despejadas. Desta forma, sua qualidade pode ser alterada pelo acúmulo de poluentes atmosféricos, uso de agrotóxicos/fertilizantes, rejeitos sólidos, materiais tóxicos e até radioativos. Quando o poluente chega à superfície do solo, ele poderá ser adsorvido; carreado pelo vento ou pelas águas de escoamento ou, até mesmo, ser lixiviado por águas de infiltração, chegando aos horizontes inferiores e atingindo o lençol freático. Uma vez que as águas subterrâneas são atingidas, esses contaminantes podem ser levados a outras regiões (CETESB, 2020).

A disposição do CONAMA (2009) informa que as propriedades químicas do solo, como $\mathrm{pH}$, teor de nutrientes, capacidade de troca iônica, condutividade elétrica e matéria orgânica, concomitantemente às atividades biológicas, são responsáveis pela adsorção, fixação química, oxidação e neutralização desses poluentes.

\section{OS AGROTÓXICOS E A CULTURA DO TOMATE}

O tomate é um fruto nativo da América do Sul. Dados históricos indicam que há mais de 100 anos o tomate já era cultivado pelos incas e astecas em regiões elevadas do 
Peru e do México. Os primeiros países a cultivarem o produto foram Peru, México, Bolívia, Equador e Chile, segundo (CURRENCE, 2013). Os maiores produtores mundiais de tomate da atualidade são: China, Brasil Estados Unidos, Índia, Turquia, Egito, Itália, Irã, Espanha e México, segundo o relatório FAOSTAT (2018).

Dados do IBGE (EPAG, 2019), mostram que o Brasil produziu 4.084.910 toneladas em 2018 e que, em janeiro de 2019, a produção foi de 4.333.609 toneladas do fruto. As regiões de maior produção em 2018 foram: sudeste com 1.689 .558 toneladas (São Paulo produziu 811.100 ton) e centro-oeste, com 1.369.014 toneladas (Goiás produziu 1.334.500 ton).

Para Junior (2019), não existe tomateiro resistente à maioria das pragas e doenças. Por isso, a maneira mais comum de controlar essas infestações continua sendo a aplicação de fungicidas e de inseticidas, o que provoca risco de contaminação dos trabalhadores envolvidos, resíduos de agrotóxicos nos frutos, impactos no meio ambiente e elevação dos custos.

No Brasil, as referências da ANVISA (2018) autorizam cerca de 500 princípios ativos com finalidades de uso agrícola, domissanitário, não agrícola, ambientes aquáticos e conservantes de madeira. Desse quantitativo, 119 agrotóxicos são autorizados para aplicação na plantação do tomate. Um mesmo princípio ativo pode ser comercializado sob a rotulação de muitas formulações e nomes comerciais, sendo comum a mistura contendo mais de um princípio em um mesmo produto.

Carvalho (2017) em sua pesquisa, cita que os agrotóxicos são utilizados na plantação do tomate com o objetivo de combater prioritariamente a mosca branca, fungos da requeima[8], talo oco, murcha bacteriana, minador das folhas, broca grande dos frutos, pinta preta, pinta bacteriana e broca pequena dos frutos. Segundo essa pesquisa, para combater tais pragas e doenças são necessárias diversas aplicações de agrotóxicos. O autor cita que os produtores fizeram referência a 53 produtos marcas comerciais diferentes e uma média de 12 tipos de agrotóxicos por lavoura. Os 
inseticidas e fungicidas são os produtos mais empregados pelo agricultor na plantação do tomate, devido a doença denominada requeima, segundo a esta pesquisa.

\section{DESTINO DOS AGROTÓXICOS NO MEIO AMBIENTE}

O emprego de agrotóxicos na cultura convencional do tomate traz constantes preocupações devido aos danos gerados ao meio ambiente, sobretudo nos meios bióticos e abióticos. Além disso, uma série de efeitos é observada entre os trabalhadores do campo: fraqueza, náuseas, tonteira, cânceres, lesões hepáticas, alergias, entre outros. Desta forma torna-se muito importante a análise dos frutos, solo e água com a finalidade de quantificação para verificar se estes se encontram dentro dos limites máximos de resíduos (LMR ${ }^{[9]}$ ) autorizados pela Anvisa, segundo (RIBAS e MATSUMURA, 2009).

\section{DESENVOLVIMENTO}

\section{METODOLOGIA - PREMISSAS, HIPÓTESES E ETAPAS DO TRABALHO}

\section{PREMISSAS}

Este trabalho partiu das seguintes premissas:

- Não existe tomateiro resistente à maioria das pragas e doenças;

- O solo é o destino final de grande parte dos resíduos da aplicação de agrotóxicos nos métodos de plantio em que esses são aplicados;

- Há provável contaminação dos solos pela aplicação de agrotóxicos nas plantações agrícolas que pode gerar impactos ambientais nos meios bióticos e abióticos; 


\section{HIPÓTESES}

- Os agrotóxicos em uso na agricultura brasileira não são ambientalmente amigáveis no que diz respeito à contaminação do fruto e do solo;

- O plantio convencional com o uso intensivo de agrotóxicos, pode não ser ambientalmente amigável;

- Existe uma lacuna na agricultura, que é a ausência de um método validado e confiável para determinação e quantificação de agrotóxicos em solo, segundo os limites máximos de resíduos (LMR) especificados pela Anvisa;

- É possível utilizar agrotóxico no plantio e manter os frutos em níveis de concentração que atendam às exigências da legislação vigente no país;

O conhecimento de resíduos e contaminantes no solo é importante para o desenvolvimento de ações para melhorar o manuseio e o controle na produção agrícola para reduzir tais contaminantes.

\section{ETAPAS DO TRABALHO}

a) Realização de pesquisa: aplicação de questionário mix de respostas abertas e múltiplas escolhas. Essa pesquisa foi aplicada junto aos plantadores das áreas em que as amostras seriam coletadas e possibilitou o levantamento dos principais produtos utilizados em suas plantações. Estes agrotóxicos foram identificados como "Agrotóxicos-Foco" do trabalho.

b) Coleta das amostras de solo em áreas de três tipos de cultivo: convencional; orgânico e sustentável;

c) Coleta das amostras de ensaio em branco de referência - Amostras dos solos que não foram submetidos a nenhum tratamento com os agrotóxicos de interesse neste trabalho;

d) Caracterização do solo quanto à textura, fertilidade e composição química - Etapa realizada em parceria nos laboratórios da EMBRAPA SOLOS-RJ; 
e) Preparo das amostras para a extração - as amostras precisam estar com granulometria de 30 mesh (terra fina), adequada à extração química - etapa realizada nos laboratórios da EMBRAPA SOLOS-RJ;

f) Análise das amostras reais de solos coletadas no campo.

Esta etapa dividiu-se em:

- Caracterização do Solo - Realizada nos Laboratórios da EMBRAPA SOLOS;

- Determinação de matéria orgânica - Etapa realizada nos Laboratórios da EMBRAPA SOLOS;

- Análise Química para determinação de micro-nutrientes dos solos - Etapa realizada no Laboratório FERTIMOVEL (EMBRAPA SOLOS);

- Extração, Clean-up, adequação e otimização do Método QuEChERS para determinação de resíduos de agrotóxicos em amostras de solos, através da Cromatografia Líquida de Ultrarresolução acoplada à Espectrometria de Massas Sequencial - Etapa Realizada nos laboratórios do INCQS - FIOCRUZ.

g) Cálculos, avaliação estatística e plotagem dos resultados obtidos.

\section{AGROTÓXICOS SELECIONADOS PARA O ESTUDO}

$\mathrm{Na}$ pesquisa realizada com os plantadores observou-se que os principais produtos utilizados em suas plantações foram: Abamectina, Acibenzolar-S-Metílico, Azoxistrobina, Ciromazina, Diafentiuron, Mandipropamida, Pimetrozina, Tiametoxan. Desta forma, optou-se por adaptar o método analítico, inicialmente, para a determinação de resíduos desses "agrotóxicos-foco" em solo proveniente do cultivo do tomate. A pesquisa foi realizada com os plantadores da região norte fluminense (Rio de Janeiro), grande produtora de tomate distribuído pelos três sistemas de cultivo estudados neste trabalho (convencional, orgânico e sustentável). 


\section{O MÉTODO DE EXTRAÇÃO (QUECHERS)}

O método de extração QuEChERS (do inglês Quick, Easy, Cheap, Effective, Rugged and Safe) pesquisado e levado à comunidade científica por ANASTASSIADES et al. (2003), tem por objetivo suprimir as limitações práticas dos métodos de extração multirresíduos existentes até então. O método apresenta como principais diferenciais, o fato de ser um rápido, fácil, econômico, efetivo, robusto e seguro, como o próprio nome QuEChERS abrevia. Diéz et al. (2006) ressaltam que esse método foi desenvolvido para amostras que apresentam mais de $75 \%$ de água.

\section{LEVANTAMENTO BIBLIOGRÁFICO SOBRE O MÉTODO QUECHERS} ALIADO À CROMATOGRAFIA PARA DETERMINAÇÃO DE
AGROTÓXICOS - EVOLUÇÃO CRONOLÓGICA

Lesueur et al. (2008) pesquisaram alterações para QuEChERS em análise de 105 agrotóxicos por cromatografia gasosa acoplada ao espectrômetro de massas (CGEM) e 46 agrotóxicos por Cromatografia Líquida de Ultra Desempenho acoplada à Espectrometria de Massas Sequencial (CLUE-EM/EM) após extração pelo método QuECheRS em quatro matrizes (uva, limão, cebola e tomate).

Drożdżyński et al. (2009) pesquisaram 3 inseticidas ecológicos (azadiractina, espinosade e rotenona) em amostras de solo, repolho e tomate utilizando o método QuEChERS modificado, com posterior determinação dos teores por CLUE-EM/EM.

Chen et al. (2010) realizaram modificação do método QuEChERS para determinação de procimidona em amostras de solo e alho-poró complementando o trabalho através da quantificação por GC-MS.

Rashid et al. (2010) analisaram 19 agrotóxicos do grupo dos organoclorados em solos, aplicando um método QuEChERS modificado e clean-up constituído por partição líquido-líquido com n-hexano. O procedimento foi validado para a determinação de 19 pesticidas organoclorados, hexaclorobenzeno (HCB), $\alpha-\mathrm{HCH}, \beta-\mathrm{HCH}, \gamma-\mathrm{HCH}$, 
heptacloro, epóxido de heptacloro (trans), aldrin, dieldrin, clordano (trans), clordano (cis), oxiclordano, $\alpha$-endossulfano, $\beta$-endossulfano, sulfato de endossulfano, endrin, $p, p^{\prime}-D D T, o, p^{\prime}-D D T, p, p^{\prime}-D D D$ e $p, p^{\prime}-D D E$.

Shi et al. (2010) propuseram um método QuEChERS modificado para a análise de resíduos de oxadiargil em amostras de solo, água, arroz e palha de arroz, com quantificação por GC-ECD.

Pinto et al., (2010) pesquisaram uma versão ainda mais simplificada do método QuEChERS com objetivo de analisar três compostos organo-clorados (hexaclorobenzeno; 1,2-diclorobenzeno e clorofórmio) em amostras de solos, seguido de quantificação por GC- $\mu \mathrm{ECD}$. No trabalho, os autores utilizaram três tipos diferentes de solos: solo de jardim, com elevado grau de matéria orgânica; um Vertisol, com elevado teor de argila; e um material sedimentoso de referência certificado (solo argiloso).

Martins (2010) utilizou o método QuEChERS - determinação de resíduos de pesticidas em solo de lavoura de arroz irrigado, empregando quechers modificado com solução saturada de hisdróxido de cálcio e LC-MS/MS para a determinação de resíduos de Clomazona, Fipronil, Imazapique, Imazetapir, Propiconazol, Tiametoxam e Trifloxistrobina.

Ramos et al. (2010) desenvolveram um método QuEChERS modificado para determinação de 11 agrotóxicos em três tipos de solos (florestal, ornamental e agrícola). Uma versão modificada do método QuEChERS foi desenvolvida para a determinação dos pesticidas organofosforados (etoprofós, dimetoato, diazinon, malaoxon, clorpirifós-metil, fenitrion, malation, clorpirifós, fenamifós e fosmet) e de um pesticida da classe das tiadiazinas (buprofezina), determinando os teores por GCNPD.

Drożdżyński et al. (2011), determinaram 160 pesticidas em vinhos empregando extração dispersa em fase sólida em modo misto e CG-EM. 
Costa (2012) realizou estudo do método QuEChERS para determinação multirresíduo de agrotóxicos em pêssego em calda. Os LOQs dos agrotóxicos neste estudo variaram entre 1,0 e10,0 $\mathrm{gg}_{\mathrm{gg}}{ }^{-1} \mathrm{e}$ baseou-se na curva para monitorar o desempenho do método e a linearidade. Segundo o autor, as curvas analíticas apresentaram valores de $r$ maiores que 0,99 ; com valores de recuperação para o pêssego em calda drenado entre 83,4 a $120,4 \%$ com RSD inferiores a $14,9 \%$ para a maioria dos analitos, e de 68,6 a $124,6 \%$ com RSD inferiores a $19,8 \%$.

Estudos de Tsipi et al., (2015) abordam a quantificação de resíduos dos metabólitos de 2,4-D, por cromatografias líquida e gasosa acopladas ao espectrômetro de massas.

Ramos et al. (2016) citam que o método QuEChERS foi utilizado apenas 8 vezes na extração de agrotóxicos em solos, e que na maioria dos casos, foi aplicada a cromatografia gasosa com detecção por Espectrometria de Massas (CG-EM), exceto em três casos, onde foram aplicados cromatografia gasosa com detectores por Captura de Elétrons (CG-DCE); Nitrogênio e Fósforo (CG-DNF) e Micro Detecção por Captura de Elétrons (CG-D $\mu \mathrm{CE})$.

Dong et al. (2017) determinaram resíduos de metaflumizona em amostras de solo e repolho, aplicando o método QuEChERS. Os autores relatam que foram obtidos valores de recuperação entre 77,6 e $87,9 \%$ para metaflumizona em solos e repolho, com desvio padrão relativo (RSD) de 3,5 e 7,9\%. Os valores de LOD e LOQ do método para as mesmas amostras foram de $0,001 \mathrm{mg} \cdot \mathrm{kg}^{-1}$ e $0,004 \mathrm{mg} \cdot \mathrm{kg}^{-1}$ respectivamente.

Segundo Iglesias (2016), o processo de acoplamento da Cromatografia Líquida à Espectrometria de Massas ocorreu muito lentamente, em função da incompatibilidade entre as altas vazões utilizadas peça HPLC que tornava difícil o carreamento do eluente da coluna cromatográfica diretamente para 0 interior da fonte do espectrômetro, que funciona em alto vácuo. Resolvidas essas dificuldades, a Cromatografia Líquida com interface de acoplamento à Espectrometria de Massas 
(LC-MS) tem se difundido cada vez mais como uma excelente técnica para determinação de resíduos de analitos diversos.

Ogihara (2018), empregou o método QuEChERS e a cromatografia líquida de ultra eficiência acoplada à espectrometria de massas sequencial na determinação de multirresíduos de agrotóxicos em solo. Em seu trabalho foram avaliadas as três versões do método QuEChERS, "Original", tampão "Acetato" e tampão "Citrato", na ausência e na presença da etapa clean up na extração de agrotóxicos do solo e a UHPLC-MS/MS com adição de padrão interno de Trifenilfosfato na quantificação e confirmação dos mesmos. O método desenvolvido teve por objetivo correlacionar determinadas propriedades físico-químicas de 20 agrotóxicos selecionados pela autora com seus respectivos tempos de dissipação dos mesmos no ambiente em presença e ausência de luz.

Todas essas pesquisas acabaram por resultar na conclusão de que os métodos clássicos para a determinação de agrotóxicos em solos não apresentam boa relação custo-benefício, pois são procedimentos que exigem muitos passos, geralmente, baseados na extração exaustiva da matriz, com etapas posteriores de clean-up para a remoção dos materiais co-extraídos, antes da análise instrumental.

\section{ESCOLHA DAS AMOSTRAS PARA A ETAPA DE VALIDAÇÃO}

As amostras utilizadas para etapa de validação foram as do solo branco que não foram submetidas a nenhum tratamento com agrotóxicos antes e durante a plantação. Após a determinação cromatográfica estas amostras apresentaram-se isentas dos agrotóxicos, não manifestando sinal cromatográfico em tempos de retenção semelhante aos dos compostos de interesse analítico.

O solo utilizado nos estudos foi classificado como Planossolo Hidromórfico Eutrófico Arênico, pertencente à unidade de mapeamento do município de Tanguá, no município do Rio de Janeiro. A região apresenta relevo plano a suavemente ondulado com substrato de sedimentos aluviais recentes. 
As propriedades físico-químicas deste solo são: $\mathrm{pH}$ água (1:1) $=4,8 ; \mathrm{P}=6,0 \mathrm{mg} \mathrm{L}^{-1}$; $\mathrm{K}=120 \mathrm{mg} \mathrm{L}^{-1} ;$ argila $=26 \%$; M.O. $=2,3 \% ; \mathrm{Ca}=5,0 \mathrm{cmolc} \mathrm{L}^{-1} ; \mathrm{Mg}=2,0 \mathrm{cmolc} \mathrm{L}^{-1} ; \mathrm{Al}$ $=1,7 \mathrm{cmolc} \mathrm{L}^{-1}$ e índice SMP 5,1

\section{REAGENTES, SOLVENTES E GASES}

Acetona P.A.; Acetonitrila - UHPLC; Sulfato de Magnésio Anidro; Cloreto de sódio P.A.; PSA - UHPLC; Água destilada; Água ultrapura, purificada em sistema Milli-QPlus; Ar sintético 99,9\% de pureza; C18 - Cartuchos para SPE; Cloreto de sódio; Diclorometano - Ultra Resi-Analyzed; Etanol- UV-IR-HPLC; Extran neutro; ás argônio, analítico, utilizado como gás de colisão no sistema CLUE-MS/MS; Gás nitrogênio, utilizado como gás de dessolvatação na fonte de electrospray; Metanol- UV-IR-HPLC; Sorvente Bondesil PSA, com tamanho de partícula de $50 \mu \mathrm{m}$;

\section{EQUIPAMENTOS}

Agitador Marconi Modelo M227; Estufa Fanem Modelo F 330; Agitador Vortex IKA Modelo MS 3 Digital; Balança analítica de precisão; Metler Toledo; Modelo XP205; no de série B018030980; Balança analítica de precisão; SARTORIUS Modelo SARTORIUS - oㅡ de série 71205517; Centrífuga; Eppendorf, Modelo 5810R; Micropipetadores automáticos Eppendorf com capacidade variável; $\mathrm{pH}$ metro Metler Toledo S 220; Sistema de purificação de água Milli-Q fabricado pela MilliPore; Cromatógrafo líquido Waters Acquity Ultraperformance LC; Espectrômetro de Massas Sequencial Quatro Premier Modelo XE.

\section{OTIMIZAÇÃO DO MÉTODO - CONDIÇÕES CROMATOGRÁFICAS E DO ESPECTRÔMETRO DE MASSA}

A análise foi realizada utilizando um sistema Acquity UPLC® acoplado ao Quattro Premier XE® (Waters Corp., Ma, EUA).

Sistema Acquity UPLC ${ }^{\circledR}$ composto por uma bomba binária, amostrador automático e forno de coluna. 
A separação cromatográfica foi realizada na coluna Waters Acquity BEH UPLC $® \mathrm{C} 18$ (100 x 2,1 mm ID, 1,7 $\mu \mathrm{m}$ ). Composições da fase móvel A (formato de amônio $5 \mathrm{mM}$ + ácido fórmico a 0,01\%, pH 4,00) e fase móvel $\mathrm{B}$ (acetonitrila: fase móvel A, 95: 5), gradiente: $0-1 \mathrm{~min}(10 \% \mathrm{~B}) ; 1$ a $5,5 \mathrm{~min}(55 \% \mathrm{~B}) ; 5,5$ a $10,5 \mathrm{~min}(100 \% \mathrm{~B}) ; 12 \mathrm{~min}$ (10\% de B). A vazão utilizada foi de $0,3 \mathrm{~mL} \mathrm{~min}^{-1}$, a temperatura do forno da coluna foi de $30^{\circ} \mathrm{C}$, a temperatura do amostrador automático foi de $25^{\circ} \mathrm{C}$. O injetor foi ajustado para injeção de loop completo de $10 \mu \mathrm{L}$ e o tempo total de execução foi de $12 \mathrm{~min}$.

O espectrômetro de massa Quattro Premier $X E^{\circledR}$ foi operado com uma fonte de ionização por eletropulverização no modo positivo $\left(\mathrm{ESI}^{+}\right)$. Os parâmetros operacionais foram ajustados para as seguintes condições: tensão capilar: $3,5 \mathrm{kV}$; temperatura da fonte de íons: $120^{\circ} \mathrm{C}$, temperatura de dessolvatação: $450{ }^{\circ} \mathrm{C}$; fluxo de gás cone $\left(\mathrm{N}_{2}\right)$ : 20 L.h ${ }^{-1}$; fluxo de gás de dessolvatação $\left(N_{2}\right)$ : 500 L.h $^{-1}$; fluxo de gás de colisão ( $\mathrm{Ar}$ ): $0,15 \mathrm{~mL} \cdot \mathrm{min}^{-1}$. As tensões do cone, as energias de colisão e as transições de quantificação e confirmação para cada analito foram estabelecidas a partir da infusão direta de solução de $1 \mu \mathrm{g} \cdot \mathrm{mL}^{-1}$. A infusão de analitos foi realizada com as fases móveis A e B (1: 1), com vazão de $0,1 \mathrm{~mL} \cdot \mathrm{min}^{-1}$ no modo de varredura completa. Após o ajuste desses parâmetros, foi estabelecido o método de monitoramento de múltiplas reações (MRM), utilizado para identificação e quantificação de analitos.

A escolha da fase móvel, modo de ionização (ESI positivo), transições de quantificação e confirmação foram feitas de acordo com a literatura (Aguilera-Luiz et al., 2011; Rubensam et al., 2011) e as características químicas dos analitos. Alguns dos parâmetros usados no sistema Quattro Premier XE ${ }^{\circledR}$, como a tensão capilar; temperatura da fonte de íons; temperatura de dessolvatação, entre outras, foram estabelecidas durante a calibração do instrumento pelo fabricante. Os íons precursores de cada analito foram observados por infusão direta. Na maioria dos casos, o íon protonado $\left[\mathrm{M}^{+} \mathrm{H}\right]^{+}$foi observado. 


\section{PADRÕES ANALÍTICOS}

Os Padrões analíticos dos agrotóxicos estudados e Preparo das soluções de trabalho (soluções estoque de fortificação)

Os padrões analíticos dos agrotóxicos utilizados foram adquiridos da Empresa AccuStandart. A Tabela 1 apresenta o grau de pureza (\%) e a classe dos padrões analíticos sólidos utilizados para o desenvolvimento deste trabalho.

Tabela 1 - Padrões analíticos sólidos utilizados no trabalho

\begin{tabular}{|l|l|}
\hline AGROTÓXICO & PUREZA (\%) \\
\hline Azoxistrobina & 99,4 \\
\hline Boscalida & 95,5 \\
\hline Carbendazim & 98,7 \\
\hline Clorantraniliprole & 98,4 \\
\hline Clotianidina & 96,5 \\
\hline Diafentiuron & 99,9 \\
\hline Difenoconazol & 100 \\
\hline Dimetomorfe & 98 \\
\hline Espinetoram & 96,8 \\
\hline Espinosade & 96,6 \\
\hline Fenuron & 98 \\
\hline Imidacloprido & 99,5 \\
\hline Indoxacarbe & 97,3 \\
\hline Metalaxil M & 98 \\
\hline Metoxifenozida & 99,5 \\
\hline Tiametoxan & 100 \\
\hline
\end{tabular}

Fonte: AccuStandart in New Haven, Connecticut, USA - 2018 
Com esses padrões foi preparada a solução estoque de fortificação contendo os analitos. Esta solução tem validade de apenas um mês e deve foi cuidadosamente armazenada em frasco âmbar, com batoque e tampa de teflon à temperatura de $18^{\circ} \mathrm{C}$, em ultra-cold.

Toda a vidraria utilizada no preparo das soluções e análises, como pipetas, balões volumétricos, provetas, etc, foi devidamente calibrada e identificada para evitar erros volumétricos nas determinações.

Inicialmente foram preparados $10 \mathrm{~mL}$ de solução analítica estoque $1000 \mathrm{mg} \cdot \mathrm{L}^{-1} \mathrm{de}$ cada agrotóxico. Os padrões foram dissolvidos em Metanol 0,02\% em ácido acético glacial que são os mesmos componentes da fase móvel adotada na cromatografia líquida que analisará os compostos e as soluções- estoque foram armazenadas em frascos âmbar a temperatura de $-18 \stackrel{\circ}{ } \mathrm{C}$.

Pelo método das diluições sucessivas foram preparadas soluções analíticas individuais de cada agrotóxico em estudo, na concentração de 100 mg.L-1, com os mesmos solventes. A partir destas soluções, preparou-se uma mistura na concentração $10 \mathrm{mg} . \mathrm{L}-1$ contendo todos os agrotóxicos. A partir da solução padrão 10 mg.L-1, finalmente, preparou-se uma mistura na concentração 0,200 mg.L-1 contendo todos os agrotóxicos.

Partindo-se da mistura intermediária $1,0 \mathrm{mg} \mathrm{L}^{-1}$, foram preparadas as soluções analíticas de trabalho nas concentrações de 0,$4 ; 2,0 ; 4,0 ; 10,0 ; 20,0$ e 40,0 $\mu \mathrm{g} . \mathrm{L}^{-1}$ contendo todos os agrotóxicos em cada concentração para a confecção da curva de calibração do cromatógrafo líquido. Para injeção no sistema UHPLC-MS/MS, foram realizadas diluições na proporção 1:1 (v/v) destas soluções em fase móvel Metanol/água, de modo que as concentrações finais das soluções de trabalho avaliadas foram 0,$2 ; 1,0 ; 2,0 ; 5,0 ; 10,0$ e $20,0 \mathrm{mg} \mathrm{L}^{-1}$ para todos os agrotóxicos constituintes da solução-estoque de fortificação. As diluições das soluções analíticas em fase móvel acidificada têm como objetivo melhorar a eficiência de ionização dos analitos, melhorando o sinal cromatográfico, a forma e simetria dos picos. Estas 
soluções de trabalho foram utilizadas para o estudo da linearidade do método. Todas as soluções foram armazenadas em frascos âmbar e estocadas a $-18^{\circ} \mathrm{C}$.

\section{VALIDAÇÃO DO MÉTODO}

A parametrização adotada para a validação do método analítico consistiu na verificação do desempenho. Desta forma, parâmetros como: curva analítica e linearidade, limite de detecção, limite de quantificação, exatidão (recuperação) e precisão (repetitividade e precisão intermediária) tornaram-se referência para a obtenção de resultados confiáveis.

\section{DETERMINAÇÃO DO SOLO BRANCO DE REFERÊNCIA}

Devido à complexidade da matriz e aos baixos níveis de concentração em que os agrotóxicos se encontram no solo (ordem de ppm a ppb), o preparo da amostra foi fundamental para a obtenção de resultados confiáveis.

O passo mais difícil foi conseguir uma amostra de solo branco, isento de agrotóxicos e que pudesse servir de referência zero para os estudos. A este solo é que se pretendia fazer as contaminações com os agrotóxicos para seguir a otimização através do método QuEChERS.

Para a verificação do solo branco foi utilizada a amostra de solo codificada como A1BR05 em dois tratamentos:

\section{TRATAMENTO 01}

Em 5 tubos de centrífuga tipo Falcon de $50 \mathrm{~mL}$ foram pesados $15 \mathrm{~g}$ do solo e procedeuse o tratamento 01 , baseado no Método QuEChERS original: $15 \mathrm{~g}$ solo $+5 \mathrm{~mL} \mathrm{H}_{2} \mathrm{O}$; vortex $30 \mathrm{seg}, 1 \mathrm{~mL}$ Surrogate (Propoxur $1.0 \mu \mathrm{g} / \mathrm{mL}$ ); vortex 30 seg; 15 min espera; $15 \mathrm{~mL}$ ACN grau UHPLC; Vortex 30 seg; $6 \mathrm{~g} \mathrm{MgSO}_{4}+1,5 \mathrm{~g} \mathrm{NaCl}$; Centrifugação (7 min); extração do sobrenadante e diluição com metanol RP 1:1 para injeção no cromatógrafo líquido. 
A solução de Propoxur 1,0 $\mu \mathrm{g} \cdot \mathrm{mL}^{-1}$ (Surrogate) foi utilizada como marcador. Caso o cromatograma do branco aparecesse sem picos, era preciso assegurar que o sistema manifestava sensibilidade aos compostos, e o propoxur foi o composto que trouxe essa certeza.

\section{TRATAMENTO 02}

Foi testado ainda um tratamento com o método modificado com solução de Hidróxido de Cálcio com $\mathrm{pH}=12,6$, a fim de obter melhor backgroud das amostras com relação à matriz solo da seguinte forma:

Em 5 tubos de centrífuga tipo Falcon de $50 \mathrm{~mL}$ foram pesados $15 \mathrm{~g}$ do solo e procedeuse o tratamento 02 - $15 \mathrm{~g}$ solo $+5 \mathrm{~mL} \mathrm{H}_{2} \mathrm{O}$; vortex 30 seg; $1 \mathrm{~mL}$ Surrogate; vortex 30 seg; $5 \mathrm{~mL}$ de Solução de $\mathrm{Ca}(\mathrm{OH})_{2} \mathrm{pH}$ 12,6; 5 min espera; $15 \mathrm{~mL}$ ACN grau UHPLC; vortex $30 \mathrm{seg} ; 6 \mathrm{~g} \mathrm{MgSO}_{4}+1,5 \mathrm{~g} \mathrm{NaCl}$; centrifugação (7 min); extração do sobrenadante e diluição com metanol RP 1;1 para injeção no cromatógrafo líquido.

As amostras do solo A1BR05 mostraram-se isentas de agrotóxicos nos dois tratamentos. A partir desta etapa, a amostra A1BR05 passou a ser o solo branco de referência deste trabalho.

Os dois tratamentos anteriores foram empregados às amostras de solo batizadas com a solução de fortificação contendo os analitos de interessse.

O método foi inicialmente otimizado para extração dos agrotóxicos-foco: Azoxistrobina, Boscalida, Carbendazim, Clorantraniliprole, Clotianidina, Diafentiuron, Difenoconazol, Dimetomorfe, Espinetoram, Espinosade A, Espinosade D, Fenuron, Imidacloprido, Indoxacarbe, Metalaxil M, Metoxifenozida, Tiametoxan proveniente de amostras de solo do cultivo do tomate, seguido de determinação por UHPLC-MS/MS (Cromatografia Líquida de Ultra Desempenho), que exige que a matriz esteja limpa, minimizando ao máximo as interferências de fundo (efeito matriz - backgroud). Sendo assim, os tratamentos 1 e 2 foram os pontos de partida para extração desses agrotóxicos da matriz do solo. 
Nos dois tratamentos os extratos ficaram bastante claros. Mesmo assim, frações de cada um dos ensaios citados anteriormente foram testadas em uma etapa de cleanup dispersivo. Nessa etapa, foi testada uma extração dispersiva em fase sólida da limpeza do PSA, gerando mais 4 tratamentos, totalizando 8 ensaios diferentes.

O extrato foi filtrado através de uma membrana de PTFE e, em seguida, $1 \mathrm{~mL}$ de extrato foi transferido para um balão volumétrico, dissolvido com $1 \mathrm{~mL}$ de Metanol e esta solução final foi transferida para um frasco vial cromatográfico. A partir deste ponto, 5 microlitros de cada amostra foram injetados em duplicata no cromatógrafo líquido de ultra Desempenho acoplada ao espectrômetro de massas. Os ensaios foram feitos em duplicata e os resultados estão plotados na tabela 2

Tabela 2 - Resultados dos tratamentos 1 e 2 das análises dos agrotóxicos-foco por cromatografia líquida de ultra desempenho

\begin{tabular}{|c|c|c|c|c|}
\hline \multirow[t]{2}{*}{ Princípio Ativo } & \multicolumn{2}{|c|}{$\begin{array}{l}\text { Fator de Recuperação sem } \\
\text { clean-up (\%) }\end{array}$} & \multicolumn{2}{|c|}{$\begin{array}{l}\text { Fator de Recuperação Pós } \\
\text { Clean-up (\%) }\end{array}$} \\
\hline & $\begin{array}{l}\text { Tratamento } \\
01\end{array}$ & $\begin{array}{l}\text { Tratamento } \\
02\end{array}$ & $\begin{array}{l}\text { Tratamento } \\
01\end{array}$ & $\begin{array}{l}\text { Tratamento } \\
02\end{array}$ \\
\hline Abamectina & $88 / 115$ & $65 / 70$ & 97,5 & 115 \\
\hline Diafentiuron & $43 / 37$ & $67 / 72$ & 53 & 81,2 \\
\hline Azoxistrobina & $101 / 100$ & $93 / 94$ & 162,5 & 160 \\
\hline Pimetrozina & $30 / 28$ & $81 / 75$ & 30 & 120 \\
\hline Acibenzolar-S-Metílico & $138 / 131$ & $36 / 38$ & 162 & 47,5 \\
\hline Mandipropamida & $108 / 109$ & $110 / 102$ & 180 & 162 \\
\hline Ciromazina & $60 / 61$ & $81 / 80$ & 95 & 125 \\
\hline Metomil & $108 / 116$ & $107 / 105$ & 177 & 225 \\
\hline Pimetrozina & $30 / 28$ & $81 / 75$ & 45 & 120 \\
\hline Acetamiprido & $103 / 104$ & $99 / 103$ & 167 & 155 \\
\hline Buprofezina & $98 / 97$ & $96 / 96$ & 167 & 166 \\
\hline Lucifenuron & $68 / 67$ & $64 / 63$ & - & - \\
\hline
\end{tabular}


Tiametoxan

$104 / 98$

$70 / 69$

165

112

Fonte: Elaboração dos autores

O tratamento com solução de hidróxido de cálcio $(\mathrm{pH}=12,3)$ apresentou melhores fatores de recuperação para a maioria dos analitos, exceto para o Acibenzolar-SMetílico que não teve boa recuperação em nenhum dos tratamentos. Possivelmente isso se deva a metilação da estrutura do composto sulfurado, que dificulta a sua extração em acetonitrila. Desta forma, a validação seguiu tomando por base o tratamento 02 .

\section{ENSAIOS DE FORTIFICAÇÃO PARA AVALIAÇÃO DA EXATIDÃO DO MÉTODO}

Para o estudo da exatidão deste método analítico, foram realizados ensaios de fortificação e com o objetivo de verificar o fator de recuperação dos compostos em estudo. Assim, foram realizadas cinco fortificações das amostras "branco de referência" em dois níveis diferentes de concentração, totalizando 10 ensaios.

Cada nível de fortificação foi injetado duas vezes, totalizando um $n=10$ (5 extrações $x 2$ injeções).

Para o procedimento de extração do método QuEChERS modificado, pesou-se 15,00 $\mathrm{g}$ de solo homogêneo em tubos de polipropileno (tipo Falcon), com tampa rosqueada (capacidade $50 \mathrm{~mL}$ ). Em seguida, umectou-se cada amostra com $5 \mathrm{~mL}$ de água Milli$Q$ e agitou-se, vigorosamente, por 30 segundos em Vortex. Adicionou a fortificação nos dois níveis, empregando-se pipetas calibradas de $0,5 \mathrm{~mL}$ e $1,0 \mathrm{~mL}$, nas concentrações: de $0,200 \mu \mathrm{g} \cdot \mathrm{mL}^{-1}$ para todos os agrotóxicos contidos na solução de fortificação.

Após fortificação, as amostras foram homogeneizadas por meio de agitação em vortex por 30 segundos e mantidas a $20 \stackrel{\circ}{\circ} \mathrm{C}$ por 15 minutos. Pesquisas de PINTO et al. (2010), indicam que é fundamental que haja tempo suficiente da amostra com os analitos para 
que o solvente evapore e, desta forma, haja maior interação entre os compostos e a matriz. Segundo o autor, essa etapa aproxima o ensaio da realidade de interação que ocorre com as amostras em campo.

Em seguida, com auxílio de uma pipeta volumétrica, $5 \mathrm{~mL}$ de solução saturada de hidróxido de cálcio pH 12,3, em cada tubo, e após fechá-los efetuou-se agitação em vortex por 30 segundos. Deixando-se reagir por 10 minutos, em repouso. Depois, processou-se a adição de $15 \mathrm{~mL}$ de acetonitrila grau Lichrosolv (para análise de resíduos) em cada tudo e agitou-se novamente por 30 segundos.

Adicionou-se 1,5 de cloreto de sódio ( $\mathrm{NaCl}$ ) e 6,0 g de $\mathrm{MgSO}_{4}$ (sulfato de magnésio anidro) em cada tudo e agitou-se por mais 30 segundos em vortex, para que se obtivesse maior interação possível entre o extrato líquido e os reagentes sólidos. Por fim, os tubos foram levados à centrifugação por 7 minutos a 3000 rpm.

Em vial com capacidade para $2 \mathrm{~mL}$, realizou-se uma diluição na proporção 1:1 (v/v), no qual foram adicionados $1,0 \mathrm{~mL}$ do extrato obtido após o a extração e 1,0 mL de fase móvel, seguido de análise por LC-MS/MS.

Por fim, foram realizadas diluições dos extratos finais na proporção 1:1 (v/v) em fase móvel (água ultrapura). A recuperação dos compostos foi avaliada nas concentrações de 1 e $2 \mu \mathrm{g} \mathrm{kg}^{-1}$ de solo para todos os agrotóxicos constantes da solução de fortificação.

Os resultados de recuperação foram interessantes nos dois tratamentos. Entretanto, o tratamento 02 mostrou-se mais eficaz na extração de maior número dos agrotóxicos, com recuperações na faixa de 64 a 110\%, excetuando-se o Acibenzolar-S-Metílico, cujas recuperações foram mais expressivas no tratamento 1.

Os resultados das experiências para avaliar o melhor método de extração e limpeza são mostrados na Tabela 1 - Ensaios realizados para a otimização da etapa de extração. 
A etapa de clean-up não demonstrou melhoras significativas nos resultados. Desta forma, optou-se por seguir para etapa de validação utilizando o tratamento 02 sem a etapa de clean-up.

\section{OTIMIZAÇÃO DO MÉTODO}

O método de extração foi otimizado de acordo com o Guia de Garantia da Qualidade Analítica. Os valores estabelecidos neste manual atendem aos requisitos da Decisão 2018/657 (European Comission/SANTE, 2018).

Os seguintes parâmetros foram avaliados: seletividade; efeito matriz; linearidade; recuperação; limite de detecção (LOD); limite de quantificação (LOQ) e repetibilidade. Os cálculos foram realizados pelos softwares MassLynx® e Microsoft Excel®. No método proposto, a seletividade foi avaliada através da análise de cinco repetições dos extratos amostrais de solos de tomateiro. A avaliação da linearidade envolveu a plotagem de uma curva analítica de solvente a partir da solução de trabalho MIX 1 contendo os 295 analitos, com cinco pontos correspondentes a 0, 0,5, 1,0, 1,5 e 2,0 vezes o LMR estabelecido para cada analito. $O$ teste de Cochran foi utilizado para avaliar a homogeneidade das variações obtidas para cada nível de concentração. Os dados de calibração foram avaliados por regressão linear comum em caso de homoscedasticidade ou regressão linear ponderada em caso de heterocedasticidade.

Para a extração das amostras foi utilizado o mesmo solo de referência dos ensaios iniciais. No método proposto, a seletividade foi avaliada através da análise de cinco repetições dos extratos amostrais do solo da plantação de tomate. Em 10 tubos de centrífuga, tipo Falcon, foram pesados $15 \mathrm{~g}$ e foi adicionado $1 \mathrm{~mL}$ da solução de trabalho. Nos tubos numerados de 1 e 5 adicionou-se $1 \mathrm{~mL}$ da solução de fortificação com soluções de trabalho de nível 1 em cada tubo, e nos tubos numerados de 6 a 10, adicionou-se $1 \mathrm{~mL}$ da solução de fortificação com soluções de trabalho de nível 2 em cada tubo, além de um tubo com ensaio em branco, sem fortificação, com controle de qualidade propoxur. Os 11 tubos receberam todas as etapas que foram utilizadas no tratamento 2 , e posteriormente, $1 \mathrm{~mL}$ do extrato foi transferido para um frasco e 
aplicou-se $1 \mathrm{~mL}$ de $\mathrm{MeOH}$ (componente da fase móvel). Depois, foi realizada a injeção de $5 \mu \mathrm{L}$ no Cromatógrafo Líquido de Ultra Desempenho acoplado ao espectrômetro de massas sequencial, nas mesmas condições adotadas para os ensaios 3 e 4 do tratamento 2. Cada amostra foi injetada em quintuplicata, conforme preconizado no Guia Sante (2018) para validação de métodos cromatográficos.

O efeito da matriz foi avaliado comparando a inclinação da curva analítica no extrato da matriz com a inclinação da curva analítica no solvente, através do teste $\mathrm{F}$ (Fisher Snedecor). Em seguida, o teste $t$ de Student foi aplicado para determinar a equivalência estatística entre as inclinações das curvas analíticas no solvente e na matriz.

O LOD e LOQ foram calculados pela relação sinal / ruído do equipamento. LOD foi a concentração equivalente a três vezes o ruído e $L O Q$ foi a concentração equivalente a seis vezes o ruído. A recuperação e repetibilidade do método foram realizadas com amostras de solos com picos de dois níveis: 0,5 a 1,0 equivalente a 5 vezes o LMR de cada analito, com cinco repetições para cada nível. A recuperação média e o desvio padrão relativo (RSD) foram calculados para cada nível. Análise de amostras. As amostras de campo foram gentilmente fornecidas pelos produtores do estado do Rio de Janeiro, Brasil, foram analisadas pelo método validado.

O método QuEChERS modificado com hidróxido de cálcio apresentou melhores resultados de recuperação que o método QuEChERS original para a maioria dos analitos, principalmente para Abamectina, Acetamiprido, Azoxistrobina, Buprofezina, Diafentiuron, Mandipropamida, Pimetrozina, Ciromazina, Metomil, Pimetrozina, Lucifenuron e Tiametoxan. Com o método modificado (método QuEChERS com Ca $\left.(\mathrm{OH})_{2}\right)$, os valores de recuperação obtidos situaram-se dentro da faixa aceitável de 70-120\% (ANVISA, 2018). O tratamento 2 teve apenas um resultado de recuperação fora da faixa aceitável de 80-110\%, Acibenzolar-S-Metílico (37\%). A etapa de clean up com SPE dispersivo não promoveu melhorias significativas nas recuperações. $A$ etapa de limpeza da SPE acabou não sendo necessária porque o primeiro extrato 
obtido foi claro e apresentou recuperações aceitáveis para os compostos de interesse, como apresentado na Tabela 2.

Portanto, o método de extração escolhido para seguir no processo de validação foi o método baseado no tratamento 2 (QuEChERS com hidróxido de cálcio) sem a etapa de clean up por SPE, usando $\mathrm{MgSO}_{4}$, PSA e C18.

A exatidão foi calculada através da Equação a seguir e foi expressa em percentagem de recuperação (INMETRO, 2007):

$$
R(\%)=\frac{C_{1} \cdot C_{2}}{C_{3}} \times 100
$$

onde:

$\mathrm{C}_{1}=$ Concentração determinada na amostra fortificada;

$\mathrm{C}_{2}=$ Concentração determinada na amostra não fortificada;

$\mathrm{C}_{3}=$ Concentração usada para fortificação.

Não houve interferência nos mesmos $\mathrm{m} / \mathrm{z}$ e tempo de retenção dos analitos nas cinco repetições realizadas com o extrato de matriz. Assim, foi possível obter-se a seletividade do método. A Planilha para avaliação da curva analítica - Validação método multirresíduos por CLUE-EM/EM é aprresentada na Figura 1. 
Figura 3 - Dados sobre a avaliação da curva analítica - Validação método multirresíduos por CLUE-EM/EM

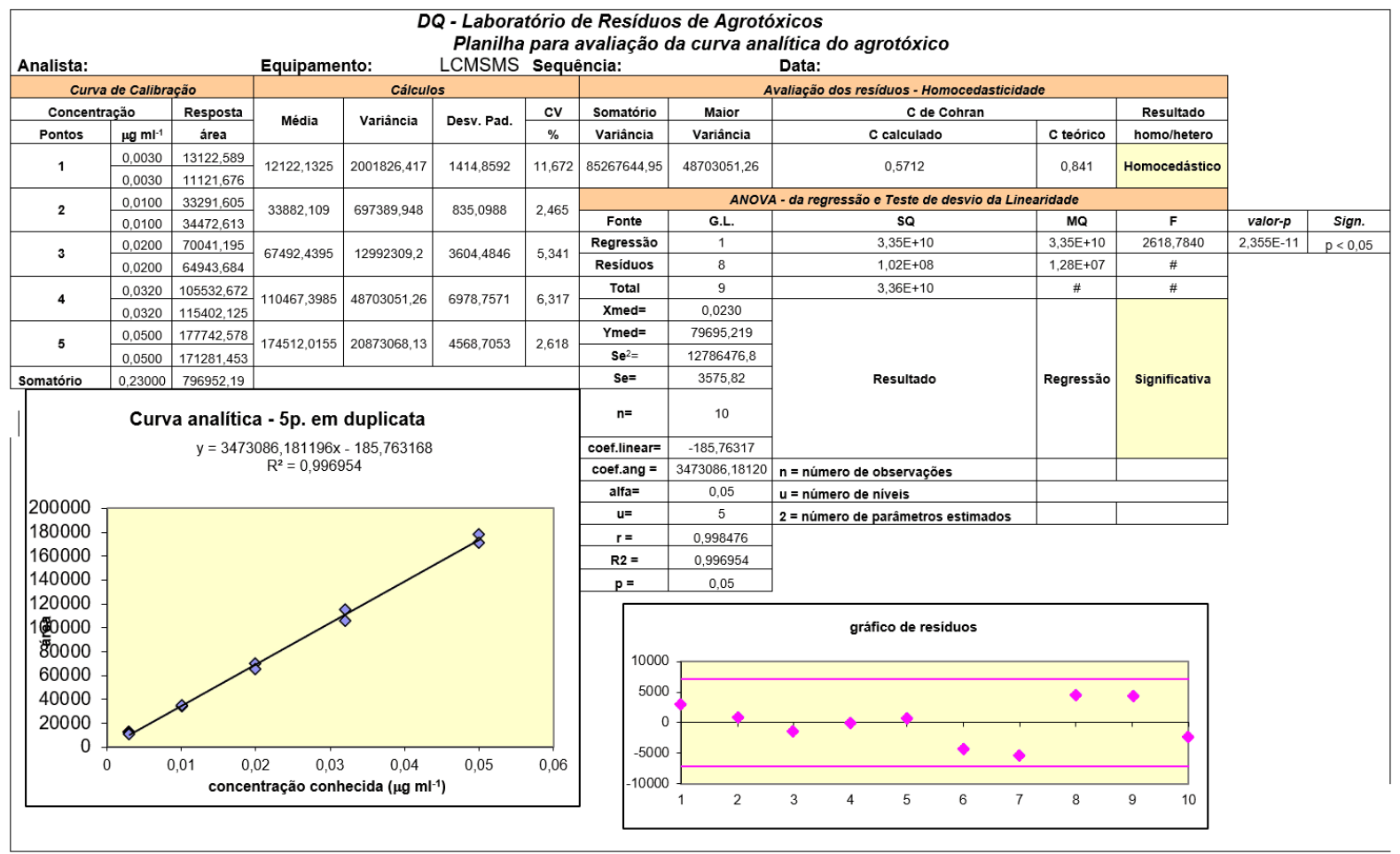

Fonte: CARDOSO et al (2010) - Software MassLynx®

As características de desempenho do método otimizado, a faixa de trabalho, os valores dos Coeficientes de correlação $(r)$ e de determinação $\left(R^{2}\right)$ para curvas analíticas obtidos para cada analito são mostrados na Tabela 3.

Tabela 3 - Resumo dos resultados da avaliação - Coeficientes de correlação ( $r$ ) e de determinação (R2)

\begin{tabular}{|c|c|c|}
\hline Substância & $\begin{array}{l}\text { VALIDAÇÃO } \\
\text { ANALÍTICA }\end{array}$ & DA CURVA \\
\hline & $r$ & $\mathrm{R}^{2}$ \\
\hline Azoxistrobina & 0,9995 & 0,9989 \\
\hline Boscalida & 0,9982 & 0,9964 \\
\hline Carbendazim & 0,9996 & 0,9993 \\
\hline
\end{tabular}

Disponível em: https://www.nucleodoconhecimento.com.br/engenharia-ambiental/metodo$\underline{\text { multirresiduos }}$ 


\begin{tabular}{|l|l|l|}
\hline Clorantraniliprole & 0,9996 & 0,9991 \\
\hline Clotianidina & 0,9963 & 0,9925 \\
\hline Diafentiuron & 0,9993 & 0,9986 \\
\hline Difenoconazol & 0,9988 & 0,9975 \\
\hline Dimetomorfe & 0,9991 & 0,9982 \\
\hline Espinetoram & 0,9973 & 0,9947 \\
\hline Espinosade & 0,9987 & 0,9974 \\
\hline Fenuron & 0,9986 & 0,9973 \\
\hline Imidacloprido & 0,9995 & 0,9990 \\
\hline Indoxacarbe & 0,9981 & 0,9961 \\
\hline Metalaxil M & 0,9998 & 0,9997 \\
\hline Metoxifenozida & 0,9969 & 0,9939 \\
\hline
\end{tabular}

Fonte: Elaboração dos autores

O efeito matriz não foi avaliado para a validação do solo, sendo considerado significante para todos os agrotóxicos estudados.

Todas as substâncias analisadas apresentaram um comportamento homocedástico no intervalo de trabalho de 0,0032 a $0,0500 \mu \mathrm{g} / \mathrm{mL}$.

Observa-se que para a maioria dos analitos, os coeficientes de determinação $\left(\mathrm{r}^{2}\right)$ foram próximos de um, mostrando boa linearidade, indicando um perfil de dispersão homoscedástica (variação constante de erros experimentais para diferentes observações) para a maioria dos analitos, permitindo que as curvas padrão fossem avaliadas por regressão linear usando o método dos mínimos quadrados ordinários. Os ajustes lineares ponderados $(1 / \mathrm{x})$ foram confeccionados através do software MassLynx®. Os valores de Student-t calculados para o efeito matriz situaram-se dentro dos valores exigidos pelo Guia SANTE para a maioria dos analitos. Assim, a curva no extrato da matriz foi utilizada para quantificar as amostras, incluindo analitos em que não foi observado o efeito matriz. Os valores obtidos para LOD e LOQ, bem como a razão sinal/ruído (Tabela 4) satisfizeram aos critérios estabelecidos pela 
Agência Nacional de Vigilância Sanitária (ANVISA, 2018) para esses analitos, confirmando que o método otimizado é adequado para atender à legislação vigente no Brasil. Entretanto, para atender à legislação europeia, o LOD e o LOQ obtidos necessitam ser revistos, pois estão muito próximos do nível máximo estabelecido (EUROPEAN COMISSION, 2018).

Tabela 4 - Substâncias validadas na matriz solo, com os respectivos limites de quantificação e a razão sinal/ruído correspondente

\begin{tabular}{|l|l|l|}
\hline Substância & $\begin{array}{l}\text { VALIDAÇÃO } \\
\text { ANALíTICA }\end{array}$ & \multicolumn{1}{c|}{ DA CURVA } \\
\cline { 2 - 3 } & LOQ $(\mathrm{mg} / \mathrm{kg})$ & Razão Sinal/Ruído \\
\hline Azoxistrobina & 0,0066 & 538,39 \\
\hline Boscalida & 0,0076 & 30,11 \\
\hline Carbendazim & 0,0055 & 166,53 \\
\hline Clorantraniliprole & 0,0075 & 276,84 \\
\hline Clotianidina & 0,0064 & 496.4 \\
\hline Diafentiuron & 0,0038 & 72,37 \\
\hline Difenoconazol & 0,0077 & 38,83 \\
\hline Dimetomorfe & 0,0072 & 27,62 \\
\hline Espinetoram & 0,0074 & 10729,08 \\
\hline Espinosade & 0,0078 & 1757,72 \\
\hline Fenuron & 0,0080 & 1630,64 \\
\hline Imidacloprido & 0,0132 & 207,19 \\
\hline Indoxacarbe & 0,0062 & 171,61 \\
\hline Metalaxil M & 0,0072 & 1104,23 \\
\hline Metoxifenozida & 0,0074 & 327,56 \\
\hline
\end{tabular}

Fonte: Elaboração dos autores

Foi possível estabelecer o LOQ para as substâncias no nível de fortificação validado, pois elas apresentaram uma razão sinal/ruído maior que 10 . 


\section{EXATIDÃo (TAXA DE RECUPERAÇÃO) E PRECISÃo (REPETIBILIDADE)}

Para o estudo da taxa de recuperação e de repetibilidade a amostra de solo A1BR05, foi fortificada com diferentes volumes da solução estoque de fortificação, compondo uma mistura dos agrotóxicos de interesse, em cinco replicatas, sendo após a extração o volume de $1 \mathrm{~mL}$ retirado e diluído 1:1 com metanol $(\mathrm{MeOH})$ para posterior análise cromatográfica por UHPLC-MS/MS. Essa concentração de fortificação corresponde a concentração teórica do LQ. Cada replicata foi injetada duas vezes.

Concentrações de injeção:

- Nível 1: 0,00323 $\mu \mathrm{g} / \mathrm{mL}$ que corresponde a $0,0067 \mathrm{mg} / \mathrm{kg}$,

- Nível 2: 0,00625 $\mu \mathrm{g} / \mathrm{mL}$ que corresponde a $0,0133 \mathrm{mg} / \mathrm{kg}$,

Os resultados obtidos da exatidão - recuperação encontram-se descritos na Tabela 5.

Tabela 5 - Resultados obtidos da exatidão - Recuperação

\begin{tabular}{|l|l|l|l|l|}
\hline \multirow{2}{*}{ Substância } & \multicolumn{4}{|l|}{ VALIDAÇÃO DA CURVA ANALítICA } \\
\cline { 2 - 5 } & Nível 1 & \multicolumn{3}{l|}{ Nível 2 } \\
\cline { 2 - 5 } & Conc. $\left(\mathrm{mg} \mathrm{kg}^{-1}\right)$ & Rec. $(\%)$ & $\begin{array}{l}\text { Conc. } \mathrm{mg} \\
\left.\mathrm{kg}^{-1}\right)\end{array}$ & Rec. (\%) \\
\hline Azoxistrobina & 0,0066 & 95,4 & 0,0142 & 106,3 \\
\hline Boscalida & 0,0076 & 111,5 & 0,0149 & 110,5 \\
\hline Carbendazim & 0,0055 & 80,4 & 0,0125 & 94,2 \\
\hline Clorantraniliprole & 0,0075 & 109 & 0,0153 & 114 \\
\hline Clotianidina & 0,0062 & 92,1 & 0,0146 & 109,9 \\
\hline Diafentiuron & 0,0038 & 53,7 & 0,0042 & 32,5 \\
\hline Difenoconazol & 0,0077 & 112,2 & 0,0148 & 111 \\
\hline Dimetomorfe & 0,0072 & 105,4 & 0,015 & 111,5 \\
\hline
\end{tabular}




\begin{tabular}{|l|l|l|l|l|}
\hline Espinetoram & 0,0074 & 103,5 & 0,0149 & 111,5 \\
\hline Espinosade & 0,0078 & 114,4 & 0,0159 & 118,9 \\
\hline Fenuron & 0,008 & 115,9 & 0,016 & 119,4 \\
\hline Imidacloprido & 0,0063 & 92,1 & 0,0143 & 106,4 \\
\hline Indoxacarbe & 0,0062 & 90,3 & 0,0135 & 101 \\
\hline Metalaxil M & 0,0072 & 105,1 & 0,0155 & 116,6 \\
\hline Metoxifenozida & 0,006 & 88,1 & 0,0146 & 109,6 \\
\hline
\end{tabular}

Fonte: Elaboração dos autores

Os resultados da recuperação estão dentro da faixa aceitável (70-120\%). O método mostrou boa repetibilidade para a maioria dos compostos pesquisados, com valores de RSD inferiores a $20 \%$.

Todos os compostos estudados satisfizeram aos critérios preconizados pela European Comission (2018), exceto o agrotóxico Diafentiuron, porque não forneceu recuperação na faixa aceitável (70 \% a $120 \%$ ) em nenhum dos dois níveis, impossibilitando a sua validação.

Após a validação o método foi utilizado para a determinação quantitativa do teor de agrotóxicos em amostras de solo colhidas nas regiões onde são plantados os tomates. Os resultados obtidos estão plotados na Tabela 6 a seguir:

Tabela 6 - Resumo dos resultados das amostras reais de solo colhidas nas áreas de plantio do tomate ( $\mathrm{mg} / \mathrm{Kg}$ de solo)

\begin{tabular}{|l|l|l|l|l|l|l|l|}
\hline Agrotóxico & A1 & A2 & A3 & A4 & A5 & A6 & A7 \\
\hline 1. Azoxistrobina & 0,003 & & & 0,002 & & 0,009 & 0,06 \\
\hline 3. Boscalida & & & & & & & Traço \\
& & & & 5 & & \\
\hline
\end{tabular}

Disponível em: https://www.nucleodoconhecimento.com.br/engenharia-ambiental/metodo- 


\begin{tabular}{|c|c|c|c|c|c|c|c|c|}
\hline 4. & Carbendazim & & & & & & $\begin{array}{l}0,006 \\
5\end{array}$ & $\begin{array}{l}0,008 \\
5\end{array}$ \\
\hline 5. & Clomazona * & & & & & & & \\
\hline $\begin{array}{l}6 . \\
e\end{array}$ & Clorantraniliprol & & & & 0,036 & $\mathrm{X}$ & 0,071 & 0,223 \\
\hline 7. & Clotianidina & & & & 0,027 & & $\mathrm{X}$ & $\begin{array}{l}0,018 \\
5\end{array}$ \\
\hline 8. & Diafentiuron & & & & $\begin{array}{l}0,025 \\
5\end{array}$ & & $\mathrm{X}$ & \\
\hline 9. & Difenoconazol & 0,003 & & & 0,038 & & & $\begin{array}{l}0,028 \\
5\end{array}$ \\
\hline 10. & Dimetomorfe & & $\begin{array}{l}0,010 \\
5\end{array}$ & & 0,48 & & 0,096 & $\begin{array}{l}0,027 \\
5\end{array}$ \\
\hline 11. & Espinetoram & & & & & & $X$ & $\mathrm{X}$ \\
\hline 12. & Espinosade A & & & & & & 0,002 & \\
\hline 13. & Espinosade D & & & & & & $\mathrm{X}$ & $\mathrm{X}$ \\
\hline 14. & Fenuron & $\mathrm{X}$ & & $\begin{array}{l}\text { Traço } \\
\text { s }\end{array}$ & $\mathrm{X}$ & $\begin{array}{l}\text { Traço } \\
\text { s }\end{array}$ & $\mathrm{X}$ & $\mathrm{X}$ \\
\hline 15. & Imidacloprido & $\mathrm{X}$ & $\begin{array}{l}\text { Traço } \\
\mathrm{s}\end{array}$ & & & & 0,008 & 0,006 \\
\hline 16. & Indoxacarbe & & & & $\begin{array}{l}0,023 \\
5\end{array}$ & & $\begin{array}{l}0,001 \\
5\end{array}$ & \\
\hline & Metalaxil M & $\begin{array}{l}\text { Traço } \\
\mathrm{s}\end{array}$ & $\begin{array}{l}\text { Traço } \\
\text { s }\end{array}$ & & $\begin{array}{l}0,008 \\
5\end{array}$ & & 0,024 & 0,001 \\
\hline & Metoxifenozida & & & & $\begin{array}{l}0,141 \\
5\end{array}$ & & $\begin{array}{l}0,010 \\
5\end{array}$ & \\
\hline & Tiametoxan & & & & $\begin{array}{l}0,031 \\
5\end{array}$ & & $\begin{array}{l}0,022 \\
5\end{array}$ & $\begin{array}{l}0,025 \\
5\end{array}$ \\
\hline
\end{tabular}

Nota: A1 a A6 (Áreas de Plantio do Tomate) Fonte: Elaborada pelos autores.

Disponível em: https://www.nucleodoconhecimento.com.br/engenharia-ambiental/metodo- 
Foram encontrados resíduos dos agrotóxicos apresentados na Tabela 6. O agrotóxico fenuron foi encontrado em todas as amostras de solo, exceto nas das áreas A1 e A2. Este agrotóxico é um dos excluído ou não registrados no Brasil, como mostrado na tabela 7. Entretanto, as concentrações desse composto encontradas nas amostras foram classificadas como traços, isto é, abaixo de limite de detecção do pelo método analítico.

Com relação aos agrotóxicos azoxitrobina e carbendazim, a situação das áreas 6 e 7 é preocupante, sobretudo porque esses agrotóxicos não são autorizados pela ANVISA para aplicação no plantio do tomate, conforme apresentado na Tabela 7.

Tabela 7 - Concentrações dos agrotóxicos NÃO AUTORIZADOS para aplicação no tomate encontrados no solos analisados

\begin{tabular}{|l|l|l|l|l|l|l|}
\hline Agrotóxico & A6 & \multicolumn{5}{l|}{ A7 } \\
\cline { 2 - 8 } & $5-10$ & $10-20$ & $10-20$ & $0-5$ & $5-10$ & $10-20$ \\
\hline 1. Azoxistrobina & 0,0090 & 0,0035 & 0,006 & 0,060 & 0,012 & 0,003 \\
\hline 2. Carbendazim & 0,0065 & 0,0065 & 0,0045 & 0,0085 & 0,003 & 0,002 \\
\hline
\end{tabular}

Fonte: Elaboração dos autores

O método multirresíduos otimizado mostrou-se seletivo e preciso na faixa estudada, permitindo a análise simultânea das substâncias: Azoxistrobina, Boscalida, Carbendazim, Clorantraniliprole, Clotianidina, Difenoconazol, Dimetomorfe, Espinetoram, Espinosade A, Espinosade D, Fenuron, Imidacloprido, Indoxacarbe, Metalaxil M, Metoxifenozida, Tiametoxan, com seus respectivos limites de quantificação (LOQ), incluídos no programa oficial de monitoramento de tomate brasileiro, conforme apresentado na Tabela 8

Tabela 8 - LOQS para os agrotóxicos-foco: $\mu \mathrm{g} \mathrm{kg-1}$

\begin{tabular}{|l|l|}
\hline Agotóxico & $\mathbf{L O Q}\left(\boldsymbol{\mu g} \mathbf{~ k g}^{-1}\right)$ \\
\hline Azoxistrobina & 7,0 \\
\hline
\end{tabular}




\begin{tabular}{|l|l|}
\hline Boscalida & 7,0 \\
\hline Carbendazim & 5,0 \\
\hline Clorantraniliprole & 7,0 \\
\hline Clotianidina & 7,0 \\
\hline Diafentiuron & 7,0 \\
\hline Difenoconazol & 7,0 \\
\hline Dimetomorfe & 7,0 \\
\hline Espinetoram & 7,0 \\
\hline Espinosade & 7,0 \\
\hline Fenuron & 7,0 \\
\hline Imidacloprido & 13,0 \\
\hline Indoxacarbe & 7,0 \\
\hline Metalaxil M & 7,0 \\
\hline Metoxifenozida & 7,0 \\
\hline
\end{tabular}

Fonte: Elaboração dos autores

\section{CONSIDERAÇÕES FINAIS}

O método QuEChERS, com pequenas alterações, foi adequado para a extração multirresíduos dos analitos em solos provenientes do plantio do, com extratos claros $\mathrm{e}$ isentos de interferências. A cromatografia líquida de ultra resolução acoplada à espectrometria de massas sequencial (UPLC-MS / MS) foi adequada para a detecção e quantificação desses analitos na matriz, com valores de recuperação entre $70 \mathrm{e}$ $120 \%$ desvio padrão inferior a $20 \%$, limites de quantificação entre 7 e $13 \mu \mathrm{g} \cdot \mathrm{L}^{-1}$ e limites de quantificação entre 2 e $4 \mu \mathrm{g} \cdot \mathrm{L}^{-1}$, apropriados para atender a legislação vigente. Os resultados do teste de campo mostraram que o método é adequado para análises quantitativas de agrotóxicos avaliados em solos derivados do plantio do tomate dentro da faixa de trabalho.

O método validado está de acordo com os valores sugeridos na literatura para a análise de resíduos de pesticidas por métodos cromatográficos (EUROPEAN 
COMISSION, 2018). A determinação dos agrotóxicos em estudo por UHPLC-MS/MS foi satisfatória, permitindo a realização de uma análise qualitativa, obtida a partir de fragmentos de massa característicos de cada analito, e quantitativa, através do modo de aquisição MRM. As condições cromatográficas otimizadas para determinação por UHPLC-MS/MS permitiram a identificação e quantificação dos compostos em estudo, em um tempo de análise menor que 15 min, o que contribui com um grande ganho como ferramenta analítica e para a sociedade como um todo.

Em aspectos gerais, todas as amostras apresentaram concentrações de agrotóxicos permitidas pelas monografias da ANVISA. Porém, os resultados obtidos para o plantio convencional, apesar de estarem dentro das conformidades exigidas, são mais elevados do que os valores obtidos para os plantios do sistema sustentável e orgânico. Entretanto, serve de alerta para a presença dos agrotóxicos na mesa da sociedade.

O emprego dos agrotóxicos Azoxistrobina e Carbendazim (agrotóxicos não autorizados) para aplicação no tomate trazem preocupação concreta com algo que normalmente já era esperado, uso deliberado dos agrotóxicos para aumento da produção, independente do que preconizam as Leis.

Se por um lado é preocupante encontrar agrotóxico não autorizado nas amostras, por outro, isso demonstra que o método validado por este trabalho apresenta elevada eficácia, devido à capacidade de quantificar até mesmo agrotóxicos não autorizados para uso.

Além de conseguir resultados bastante satisfatórios para os agrotóxicos-foco, este trabalho mostrou-se capaz de determinar resíduos para 240 agrotóxicos, entre autorizados e não autorizados pela ANVISA no Brasil, com valores de coeficiente de determinação maiores que 0,99; valores de LOQ de $13 \mathrm{\mu g} \mathrm{kg}^{-1}$ para Espinosade e 7,0 $\mu \mathrm{g} \mathrm{kg}^{-1}$ para os demais pesticidas. O método apresentou boa precisão, com valores de $\mathrm{RSD}<20 \%$, e exatidão, com recuperações entre 70 e $120 \%$ para a grande maioria dos compostos analisados. 


\section{REFERÊNCIAS}

AZEVEDO, E. de - Alimentos Orgânicos: ampliando conceitos de saúde humana, ambiental e social, Livros G.Play, 2018.

ANASTASSIADES M, Lehothay S.J, Stajnbaher D, Schenck F.J. Fast and easy multiresidue method employing acetonitrile extraction/partitioning and "dispersive solid-phase extraction" for the determination of pesticide residue in produce. J. AOAC Int. 86: 412-431, 2003.

ANVISA - Agência Nacional de Vigilância Sanitária. Disponível em: $<$ <ttp://portal.anvisa.gov.br> 2014. Acesso em: 11 de outubro de 2018.

ANVISA - NOTA TÉCNICA 02/2017 - Posicionamento da Anvisa referente à Recomendação 028/2016 aprovada em Reunião Plenária do Conselho, 2017

CARDOSO, Maria Helena Wohlers Morelli; GOUVÊA, Adherlene Vieira; NÓBREGA, Armi Wanderley da; ABRANTES, Shirley de Mello Pereira - Validação de método para determinação de resíduos de agrotóxicos em tomate: uma experiência laboratorial - Ciência e Tecnologia de Alimentos, Campinas, 30 (Supl.1): 63-72, maio 2010.

CARVALHO, C.R.F., PONCIANO N.J.; SOUZA P.M. de. - Viabilidade econômica e de risco da produção de tomate no município de Cambuci/RJ, - Ciência Rural, Brasil, 2014.

CETESB (Companhia Ambiental do Estado de São Paulo) - Qualidade do solo, 2020. Disponível em: https://cetesb.sp.gov.br/solo/poluicao/ - Acessado em 09/2020.

COMMISSION DECISION No. 657/2002. Implementing Council Directive 96/23/EC concerning the performance of analytical methods and the interpretation of results. Off. J. Eur. Commun. L 221: 8-36. 
COMMISSION REGULATION (E.C). №. 1881/2006. Setting maximum levels for certain contaminants in foodstuffs. Off. J. Eur. Commun. L364: 5-24.

CONAMA - Resolução № 420/2009 - Disposição sobre critérios e valores orientadores de qualidade do solo quanto à presença de substâncias químicas e suas providências.

Disponível

em:

http://www.mma.gov.br/port/conama/legiabre.cfm?codlegi=620> Acesso em: 02 jan. 2019.

CURRENCE, T.M. Tomato breeding. I. Species, origin and botanical characters. Handbuch der Pflanzenzuchtung, p. 351-369, 2013.

DIEZ, C.; TRAAG, W. A.; ZOMMER, P.; MARINERO, P.; ATIENZA, J.; J. - Extraction and chromatographic analysis of the cadusafós nematicide, chromatography, 1131, $1135-2006$.

DONG, H.; XIAO, K. - Modified QuEChERS combined with ultra high performance liquid chromatography tandem mass spectrometry to determine seven biogenic amines in Chinese traditional condiment soy sauce - Food Chemistry, Volume 229, Pages 502-508 - Elsevier, 2017.

DROŻDŻYŃSKI, D.; KOWALSKA, J. - Rapid analysis of organic farming insecticides in soil and produce using ultra-performance liquid chromatography/tandem mass spectrometry, Springer, Talanta, 2009.

DROŻDŻYŃSKI , D.; WALORCZYK, S.; GNUSOWSKI , B - Multiresidue determination of 160 pesticides in wines employing mixed-mode dispersivesolid phase extraction and gas chromatography-tandem mass spectrometry, Volume 85, Issue 4, Talanta - Elsevier, Pages 1856-1870, 2011.

ESALQ - Simpósio de defensivos agrícolas: tópicos relevantes e principais desafios, ESALQ / 2017 
EUROPEAN COMMISSION - Directorate General for Healph and Food Safety, 2017.

EUROPEAN COMMISSION - Directorate General for Healph and Food Safety, 2018.

EMBRAPA - Manual de Procedimentos de Coleta de Amostras em Áreas Agrícolas para Análise da Qualidade Ambiental: Solo, Água e Sedimentos, 2016.

EPA (Environmental Protection Agency) - Pesticide safety for farmworkers, 2017.

FAOSTAT - Food and Agriculture Organization of the United Nations - Produtividade Mundial, 2018. Disponível em: <http://faostat.fao.org/site/340/default.aspx>. Acesso em. 28 de novembro de 2019.

FERNANDES V.C, LEHOTAY S.J, GEIS-ASTEGGIANTE L, KWON H, Mol H.G.J, van der Kamp H, MATEUS N, DOMINGUES V.F, DELERUE-Matos, C. Analysis of pesticides residues in strawberries and soils by GC-MS/MS, LC-MS/MS and two dimensional GC-time-of-flight MS comparing organic and integrated pest management farming. Food Addit Contam. 31: 262-270, 2014.

IARC. INTERNATIONAL AGENCY FOR RESEARCH ON CANCER. WHO. World Health Organization. IARC Monographs on the Evaluation of Carcinogenic Risks to Humans. 82: 183-193, 2002.

IBAMA-Ministério do Meio Ambiente: Avaliação do Potencial de Periculosidade Ambiental (PPA) de Agrotóxicos e Afins. Disponível em: Acesso em: abr. 2020.

IBGE - Indicadores / Estatística da Produção Agrícola Levantamento Sistemático da Produção Agrícola 2017-2018, 2019.

IGLESIAS, Hoshi A. - Introdução ao Acoplamento Cromatografia Líquida Espectrometria de Massas Waters Technologies do Brasil, Barueri, SP, 2016. 
INMETRO. Instituto Nacional de Metrologia, Qualidade e Tecnologia. Orientação sobre Validação de Métodos Analíticos - DOQ-CGCRE-008. Rev. 07, 28 f, jul 2018. JUNIOR, J.C.L, - Manejo integrado de pragas na cultura do tomate: uma estratégia para a redução do uso de agrotóxicos - Extensão em Foco (ISSN: 2317-9791), 2019.

LESUEUR, A. B; KNITTL P. A ; GARTNER, M. A; MENTLER A. C, FUERHACKER M.B.- Analysis of 140 pesticides from conventional farming foodstuff samples after extraction with the modified QuECheRS method - Science Direct, 2008.

MALDANER L, JARDIM ICSF. 0 estado da arte da cromatografia líquida de ultraeficiência [The art state of ultra performance liquid chromatography]. Quim Nova. 32: 214-222. Portuguese, 2009.

OGIHARA, Camila Honda - Desenvolvimento e validação de método para a determinação de multirresíduos de agrotóxicos em solo empregando o método quechers e a cromatografia líquida de ultra alta eficiência acoplada à espectrometria de massas sequencial - UNICAMP, 2018.

ORTELLI, D; COGNARD, E; JAN, P; EDDER, P. Comprehensive fast multiresidue screening of 150 veterinary drugs in milk by ultra-performance liquid chromatography coupled to time of flight mass spectrometry and Chromatography. 877: 2363-2374, 2009.

PINTO, C.G., Martín, S.H., PAVÓN, J.L.P., CORDERO, B.M., A simplified Quick, Easy, Cheap, Effective, Rugged and Safe approach for the determination of trihalomethanes and benzene, toluene, ethylbenzene and xylenes in soil matrices by fast gas chromatography with mass spectrometry detection. Anal. Chim. Acta, 689, 129-136, 2011.

PRESTES, O.D. ; Friggi, C.A.; ADAIME M.B, ZANELLA, R. QuECheRS - QuEChERSa modern method of sample preparation for multiresidue determination of 
pesticides in food by chromatographic methods coupled to mass spectrometry]. Quim Nova. 32: 1620-1634. Portuguese, 2009.

RAMOS, A. M. et al. Evaluation of a modified QuEChERS method for the extraction of pesticides from agricultural, ornamental and forestal soils. Analytical and Bioanalytical Chemistry, v. 396, p. 2307-2319, 2016.

RASHID A., NAWAZ S., BARKER H., AHMAD I., AND ASHRAF M., Development of a simple extraction and clean-up procedure for determination of organochlorine pesticides in soil using gas chromatography-tandem mass spectrometry. J. Chromatogr. A, 2010, 1217, 2933-2939

RIBAS, P. P.; MATSUMURA, A. T. S. A química dos agrotóxicos: impacto sobre a saúde e meio ambiente. Revista Liberato, 2009, 10, 14, 149-158.

RUBENSAM G, Barreto F, HOFF RB, KIST TL, PIZZOLATTO, TM. A liquid-liquid extraction procedure followed by a low temperature purification step for the analysis of macrocyclic lactones in milk by liquid chromatography-tandem mass spectrometry and fluorescence detection. Anal Chim Acta. 705:24-29, 2011.

SOBER - Revista de Economia e Sociologia Rural (RESR) - Relatório - 1 Trimestre 2019, MAPA, Brasil, 2019

TSIPI, D.; BOTITSI, H.; ECONOMOU, A. Mass Spectrometry for the Analysis of Pesticide Residues and their Metabolites. New Jersey: John Wiley \& Sons, 2015. VEIGA, Denise Piccirillo - O impacto do uso do solo na contaminação por agrotóxicos das águas superficiais de abastecimento público - USP, São Paulo, 2017 


\section{APÊNDICE - REFERÊNCIAS DE NOTA DE RODAPÉ}

8. A requeima, causada por Phytophthora infestans, é uma doença altamente agressiva na cultura do tomate, capaz de dizimar lavouras inteiras em curto espaço de tempo.

9. Parâmetro agronômico estabelecido pela ANVISA referente à recomendação 028/2016 aprovada pelo Conselho Nacional de Segurança Alimentar e Nutricional (CONSEA) - constitui um dos componentes para o cálculo da exposição e avaliação do risco que é pré-requisito para o registro ou a autorização de um agrotóxico em novas culturas.

Enviado: Dezembro, 2020.

Aprovado: Janeiro, 2021. 